\title{
Optimization of the plate drying process by electromagnetic radiation using the Green function method
}

\author{
Anatoliy Afanas'ev, Yulia Bakhracheva* \\ Volgograd State University, Institute of priority technologies, Department of Information Security, \\ 400062, Volgograd, Russian Federation
}

\begin{abstract}
An initial boundary value problem is formulated for calculating the heat-mass-energy fields in a homogeneous wet plate. To solve this problem, an original algorithm containing elements of analytical and numerical methods has been developed. In this method, solutions are written using the spatially one-dimensional Green function of the Neumann problem, which contains eigenvalues and eigenfunctions of the Sturm-Liouville boundary value problem. In comparison with the known numerical algorithms, the problems of the theory of electromagnetic drying for which its application can be effective are indicated. It is shown that the discrete supply of microwave energy significantly reduces the gradients of temperature, steam and moisture content, while reducing energy consumption by $11 \ldots 12 \%$. The probability of an undesirable spontaneous temperature increase at the end of the drying cycle is significantly reduced, and the electrical and thermal conditions of the microwave energy source are improved.
\end{abstract}

\section{Introduction}

The main difference between electromagnetic drying and convective and conductive drying, which currently produce up to $90 \%$ of dry products in the food industry, is that heat is released not on the surface of the material, but through its volume to a certain depth. This reduces energy losses and increases the drying rate without the risk of overheating the product. In addition, electromagnetic technology is characterized by: the possibility of using any atmospheres and vacuum; selectivity in the multicomponent structure of the material; flexibility and high control accuracy due to the low inertia of the process, the ability to accurately dispense energy, the presence of several control channels; saving material and labor resources, improving product quality and labor productivity, reducing harmful effects on the environment.

It is possible to fully use the advantages of electromagnetic methods of heating and drying only in the presence of mathematical models and means of their analysis, which provide a theoretical basis for the development of devices of this type and allow you to optimize their operation. Research on the process of drying by electromagnetic radiation is

* Corresponding author: bakhracheva@ volsu.ru 
given much attention in the literature on technologies in various industries. Studies of the drying process are performed using both analytical and numerical methods [1, 2].

First, this is due to the fact that the drying tasks are set as nonlinear in most cases. Secondly, in the drying problems we always have a system of several desired functions, while in the Fourier method we are talking about initial-boundary value problems for only one desired function of coordinates and time. This creates a big problem, since in General it is impossible to obtain separate equations for each of the desired functions from a system of partial differential equations [3].

To solve this problem, the idea of splitting the process by physical factors can be used [4]. In the article, a calculation scheme is proposed on the example of drying a homogeneous plate.

\section{A Mathematical Model of the Drying Plate}

The diagram of electromagnetic drying of a flat sample is shown in the figure. The condition of heat and moisture insulation of the lower surface of the plate means that the initial object of study is a plate with a thickness of $2 d$, which, due to the identity of the boundary conditions and irradiation conditions on the surfaces $x=0$ and $x=2 \mathrm{~d}$, does not have heat and moisture flows through the plane of symmetry $x=d$.

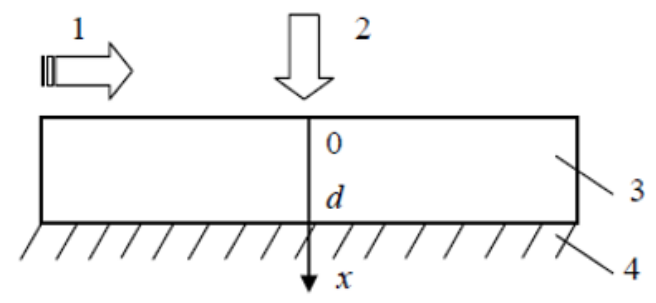

Fig. 1. Analogous to the acceleration of the pusher (a) and the radius of curvature of the cam profile (b) by the angle of its rotation: 1 - three-arc; 2-profiled on the basis of the numerical method

The material consists of a solid base (capillary-porous body) and water. Let us assume the following approximation: the plate material is assumed homogeneous; plate thickness $2 d$ is small compared with its dimensions in directions perpendicular to the axis of $x$; the density of surface heat sources $S$ caused by absorption of radiation with a small penetration depth and the heat transfer rate $Q$ and the intensity of mass transfer $J$ of the surface $x=0$ to the air weakly change along this surface, i.e., these quantities depend only on time $t$; the density of internal heat sources $W$ caused by the absorption of penetrating electromagnetic radiation depends only on the $x$ coordinate and time $t$.

In the described situation, the distribution of temperature $T$ and moisture content $U$ will depend only on $x$ and $t$, i.e. the desired functions will be $T(x, t)$ and $U(x, t)$. The initial boundary value problem for these functions will have the following form [6-8]:

$$
\begin{gathered}
c \rho \frac{\partial T}{\partial t}=k \frac{\partial^{2} T}{\partial x^{2}}+r \gamma \rho \frac{\partial U}{\partial t}+W(x, t) ; \quad 0<x<d \\
\frac{\partial U}{\partial t}=a_{\mathrm{m}} \frac{\partial^{2} U}{\partial x^{2}}+a_{\mathrm{m}} \delta \frac{\partial^{2} T}{\partial x^{2}} ; \quad 0<x<d \\
Q(t)+r(1-\gamma) \cdot J(t)=k \frac{\partial T}{\partial x}(0, t)+S(t) ; \quad x=0
\end{gathered}
$$




$$
\begin{gathered}
J(t)=a_{\mathrm{m}} \rho \frac{\partial U}{\partial x}(0, t)+a_{\mathrm{m}} \rho \delta \frac{\partial T}{\partial x}(0, \tau) ; \quad x=0 \\
\frac{\partial T}{\partial x}(d, t)=0 ; \quad \frac{\partial U}{\partial x}(d, t)=0 ; \quad x=d \\
T(x, 0)=\varphi_{T}(x) ; \quad U(x, 0)=\varphi_{U}(x) ; \quad 0 \leq x \leq d ; \quad t=0
\end{gathered}
$$

Here $c, \rho, k, \gamma, a_{\mathrm{m}}, \delta$ are the thermophysical characteristics of the material, such as specific heat capacity, density in the dry state, coefficient of thermal conductivity, evaporation criterion, moisture diffusion coefficient, relative coefficient of thermal diffusion of moisture; $r$ is the specific heat of water vaporization; $\varphi_{T}(x)$ and $\varphi_{U}(x)$ are set functions that determine the distribution of temperature and moisture content at the initial time $t=0$. The intensity of heat and mass exchange of the sample surface with the air medium $Q$ and $J$ are uniquely determined by the surface temperature $T(0, t)$ and are calculated

$$
\begin{gathered}
Q(t)=\sigma \tilde{A}\left[\left(T(0, t)+T_{1}\right)^{4}-\left(T_{\mathrm{B}}+T_{1}\right)^{4}\right]+\alpha_{\mathrm{w}}\left[T(0, t)-T_{\mathrm{B}}\right] \\
J(t)=\alpha_{\mathrm{m}}\left[P(T(0, t))-\psi \cdot P\left(T_{\mathrm{B}}\right)\right] ; \quad P(T)=6,03 \cdot 10^{-3} \cdot \exp \frac{17,3 \cdot T}{T+T_{2}}
\end{gathered}
$$

In these formulas: $\sigma$ - the Stefan-Boltzmann constant; $\tilde{A}-$ the coefficient of thermal radiation; $T_{\mathrm{B}}$ and $\psi$ - the temperature and humidity of the air beyond the boundary layer; $a_{\mathrm{w}}$ and $a_{\mathrm{m}}$ - the coefficients of heat and mass exchange of the sample surface with the air medium; $P(T)$ - a function that models the dependence of the relative partial pressure of saturated water vapor on its temperature $T$ at General normal pressure; $T_{1}=273^{\circ} \mathrm{C}$ and $\mathrm{T}_{2}=238^{\circ} \mathrm{C}-$ constants. The intensity of heat exchange $Q$, according to (7), includes heat exchange by convection and heat exchange by radiation, and the intensity of mass exchange $J$, according to (8), is taken as Dalton's law of evaporation.

\section{The Algorithm of Procedure of Splitting}

Starting from the moment $t=0$, we will solve the problem on a small interval of time $0<t<\Delta t$. Using the given initial temperature distribution $\varphi_{T}(x)$, calculate for $\mathrm{t}=0$ the second term in the right part of equation (2) (denote it as $f_{U}(x)$ ), and find using formulas (7) and (8) the intensity of heat and mass transfer $Q$ and $J$ on the surface of the plate at the same moment (denote them as $Q^{0}$ and $J^{0}$ ). Then, from the boundary conditions (3) and (4), we define at the same moment $t=0$ the normal derivatives of the fields $T$ and $U$ on the boundary $x=0$ (we denote them as $\mu_{\mathrm{T}}$ and $\mu_{\mathrm{U}}$ ). The results will be as follows:

$$
\begin{gathered}
a_{\mathrm{m}} \delta \cdot \partial^{2} T(x, 0) / \partial x^{2}=a_{\mathrm{m}} \delta \cdot d^{2} \varphi_{T}(x) / d x^{2} \equiv f_{U}(x) \\
Q(0)=\sigma \tilde{A}\left[\left(\varphi_{T}(0)+T_{1}\right)^{4}-\left(T_{\mathrm{B}}+T_{1}\right)^{4}\right]+\alpha_{\mathrm{w}}\left[\varphi_{T}(0)-T_{\mathrm{B}}\right] \equiv Q^{0} \\
J(0)=\alpha_{\mathrm{m}}\left[P\left(\varphi_{T}(0)\right)-\psi \cdot P\left(T_{\mathrm{B}}\right)\right] \equiv J^{0} \\
\partial T / \partial x(0,0)=\left[Q^{0}+r(1-\gamma) \cdot J^{0}-S(0)\right] / k \equiv \mu_{T} \\
\partial U / \partial x(0,0)=J^{0} /\left(a_{\mathrm{m}} \rho\right)-\delta \mu_{T} \equiv \mu_{U}
\end{gathered}
$$


Returning to the field $U(x, t)$ will not be taken into account in the propagation equation of moisture, (2) our small interval $0<t<\Delta t$ change in time derivative $\partial^{2} T / \partial x^{2} \quad(0<x<d)$; we also assume small changes and the derivative $\partial U / \partial x$ in the boundary condition moisture content $(x=0)$. Then to approximate the calculation of this field, we get the following initial boundary value problem:

$$
\left\{\begin{array}{l}
\partial U / \partial t=a_{\mathrm{m}} \cdot \partial^{2} U / \partial x^{2}+f_{U}(x) ; 0<x<d ; 0<t<\Delta t \\
\partial U / \partial x(0, t)=\mu_{U} ; \quad \partial U / \partial x(d, t)=0 \\
U(x, 0)=\varphi_{U}(x)
\end{array}\right.
$$

This is a Neumann problem for an inhomogeneous parabolic equation with inhomogeneous boundary and initial conditions. You can solve this problem using the Fourier method. Assuming that this problem is solved, we calculate the derivative $\partial U / \partial t$ at all points $\mathrm{x}$ for $t=0$ and denote

$$
[r \gamma \rho \cdot \partial U / \partial t(x, 0)+W(x, 0)] /(c \rho) \equiv f_{T}(x)
$$

Then, ignoring the change in time and the last two terms in the right part (1), and the derivative $\partial T / \partial x$ at the boundary $x=0$, we get the Neumann problem for finding the temperature field $T(x, t)$, similar to the one obtained above for the moisture content field:

$$
\left\{\begin{array}{l}
\partial T / \partial t=a_{\mathrm{w}} \cdot \partial^{2} T / \partial x^{2}+f_{T}(x) ; 0<x<d ; 0<t<\Delta t \\
\partial T / \partial x(0, t)=\mu_{T} ; \quad \partial T / \partial x(d, t)=0 \\
T(x, 0)=\varphi_{T}(x)
\end{array}\right.
$$

Here $a_{\mathrm{w}}=k /(c \rho)$ is the heat diffusion coefficient.

Having solved this problem using the Fourier method, we find the temperature field as a function of coordinates and time.

Thus, we have constructed a procedure for splitting the General initial-boundary value problem (1)-(8) for two functions $T$ and $U$. Direct investigation of the General initialboundary value problem by the Fourier method cannot be performed.

But for two separate problems (9) and (10), the Fourier method can already be applied. The validity of the constructed solutions $U(x, t)$ and $T(x, t)$ is limited by a small time interval $0<t<\Delta t$.

\section{Solving the Neumann Problem}

In general, the initial boundary value problems (9) and (10) can be written as follows:

$$
\left\{\begin{array}{l}
\partial Y / \partial t=a \cdot \partial^{2} Y / \partial x^{2}+f(x) ; 0<x<d ; 0<t<\Delta t \\
\partial Y / \partial x(0, t)=\mu_{1} ; \quad \partial Y / \partial x(d, t)=\mu_{2} \\
Y(x, 0)=\varphi(x)
\end{array}\right.
$$

This is a one-dimensional Neumann problem for the diffusion equation in a homogeneous medium. Here $a$ is the diffusion coefficient; $f(x)$ and $\varphi(x)$ are the given functions; $\mu_{1}$ and $\mu_{2}$ are the given numbers; $Y(x, t)$ is the desired function.

We solve this problem by using the eigenfunctions of the Sturm-Liouville problem and the apparatus of Green's functions. Based on [10], we present information from the theory of solving initial-boundary value problems that is necessary for our case. 
The solution to the original problem (11) for the $Y$ field is represented as a sum:

$$
Y=Y_{1}+Y_{2}+Y_{3}
$$

where the functions on the right side are found as solutions to other, simpler initialboundary value problems. Exactly, these problems have the same form (11), in which $f=\mu_{1}=\mu_{2}=0$ (the problem for $Y_{1}$ ); $\mu_{1}=\mu_{2}=\varphi=0$ (the problem for $Y_{2}$ ); $f=\varphi=0$ (the problem for $Y_{3}$ ) is successively assumed.

Solutions to problems for $Y_{1}$ and $Y_{2}$ look like this:

$$
Y_{1}(x, t)=\int_{0}^{d} G\left(x, t, x^{\prime}\right) \varphi\left(x^{\prime}\right) d x^{\prime} \quad Y_{2}(x, t)=\int_{0}^{t} \int_{0}^{d} G\left(x, t-\tau, x^{\prime}\right) f\left(x^{\prime}\right) d x^{\prime} d \tau
$$

In these formulas, the Green function of the original problem (11) for the y field is used. According to [11], it has the following form:

$$
G\left(x, t, x^{\prime}\right)=\frac{1}{d}+\frac{2}{d} \sum_{n=1}^{\infty} \exp \left[-\left(\frac{\pi n}{d}\right)^{2} a t\right] \cdot \cos \frac{\pi n}{d} x \cdot \cos \frac{\pi n}{d} x^{\prime}
$$

As for the problem for $Y_{3}$, its solution is sought in the form

$$
Y_{3}=\bar{Y}+\mu_{1} x+\frac{\mu_{2}-\mu_{1}}{2 d} x^{2}
$$

Here $\bar{Y}$ is the new desired function. Substituting function (15) in the Neumann problem for $Y_{3}$, we get for $\bar{Y}$

$$
\left\{\begin{array}{l}
\partial \bar{Y} / \partial t=a \cdot \partial^{2} \bar{Y} / \partial x^{2}+\bar{f} \\
\partial \bar{Y} / \partial x(0, t)=0 ; \quad \partial \bar{Y} / \partial x(d, t)=0 \\
\bar{Y}(x, 0)=\bar{\varphi}(x)
\end{array}\right.
$$

where

$$
\bar{f}=\frac{a\left(\mu_{2}-\mu_{1}\right)}{d} ; \quad \bar{\varphi}(x)=-\mu_{1} x-\frac{\mu_{2}-\mu_{1}}{2 d} x^{2}
$$

Now substitute the Green function (14) in formulas (13) and (17), and after completing the integration, write the final expressions for the functions $Y_{1}, Y_{2}$ and $Y_{3}$. Calculations should be made at a sufficiently close to $t=0$ moment $t=\Delta t$. However, as it is accepted in the theory of drying, instead of $\Delta t$, we will introduce dimensionless time into the formulas. $\Delta t^{*}=a \cdot \Delta t / d^{2}$

Here is the final result:

$$
\begin{gathered}
Y_{1}(x, \Delta t *)=\varphi_{0}+\sum_{n=1}^{\infty} \exp \left[-(\pi n)^{2} \Delta t *\right] \cdot \varphi_{n} \cdot \cos \frac{\pi n}{d} x \\
Y_{2}\left(x, \Delta t^{*}\right)=\frac{d^{2}}{a} \cdot f_{0} \cdot \Delta t^{*}+\frac{d^{2}}{a \pi^{2}} \sum_{n=1}^{\infty} \frac{1}{n^{2}}\left\{1-\exp \left[-(\pi n)^{2} \Delta t *\right]\right\} \cdot f_{n} \cdot \cos \frac{\pi n}{d} x \\
Y_{3}\left(x, \Delta t^{*}\right)=-\frac{d\left(2 \mu_{1}+\mu_{2}\right)}{6}+\mu_{1} x+\frac{\left(\mu_{2}-\mu_{1}\right)}{2 d} x^{2}+d\left(\mu_{2}-\mu_{1}\right) \Delta t^{*}+ \\
+\frac{2 d}{\pi^{2}} \sum_{n=1}^{\infty} \frac{1}{n^{2}}\left[\mu_{1}-\mu_{2}(-1)^{n}\right] \cdot \exp \left[-(\pi n)^{2} \Delta t *\right] \cdot \cos \frac{\pi n}{d} x .
\end{gathered}
$$


In these formulas $\varphi_{n}$ and $f_{n}, \mathrm{n}=0,1,2, \ldots$ are the Fourier coefficients of functions $\varphi(x)$ and $f(x)$ in their series expansions by cosines on the segment $[0, d]$. For an arbitrary function $y(x)$, this decomposition has the following form [12]:

$$
y(x)=y_{0}+\sum_{n=1}^{\infty} y_{n} \cos \frac{\pi n}{d} x ; \quad y_{0}=\frac{1}{d} \int_{0}^{d} y(x) d x ; \quad y_{n}=\frac{2}{d} \int_{0}^{d} y(x) \cdot \cos \frac{\pi n}{d} x d x .
$$

So, the solution of the Neumann problem (11) has the form (12), where the functions $Y_{1}$, $Y_{2}$ and $Y_{3}$ are found by formulas (18)-(20).

\section{Discussion}

Let's list the main steps of the calculation algorithm. The function $f_{U}(x)$ and the number $\mu_{U}$ are calculated from the given initial distributions $\varphi_{T}(x)$ and $\varphi_{U}(x)$, and the Neumann problem (9) is formulated for calculating the field $U(x, t)$. Having solved this problem, we calculate the function $f_{T}(x)$, the number $\mu_{T}$ and set the Neumann problem (10) to calculate the field $T(x, t)$. It is solved in the same way as the previous problem. Then the functions $U(x, t)$ and $T(x, t)$ are calculated at the moment $t=\Delta t$ close to $t=0$. The resulting $x$-coordinate functions are declared as initial distributions, after which the above algorithm for transitioning between neighboring layers in time is repeated.

The solution to the Neumann problem has the form

$$
U=U_{1}+U_{2}+U_{3}
$$

where the functions on the right side of this equality can be found by formulas that use higher-order approximations, where $Y_{1}, Y_{2}, Y_{3}$ should be replaced by $U_{1}, U_{2}, U_{3} ; \varphi(x), f(x)$, $a, \mu_{1}$ should be replaced by $\varphi_{U}(x), f_{U}(x), a_{\mathrm{m}}, \mu_{U}$; take $\mu_{2}=0$.

When switching to a new layer over time, the calculation is performed using formulas determined by the temperature and moisture content distributions on the previous layer over time. This makes the proposed calculation scheme fundamentally different from the standard procedures of grid methods, in which these distributions are included as arrays in a system of linear algebraic equations of high orders, which need to be solved in order to make the transition between layers in time.

Calculations performed on a sample of wood with a humidity of $60 \%$ show a high degree of uniformity of heating and a 2 -fold decrease in the reflection coefficient. Thus, the power loss on reflection can be $5 . .20 \%$. It is established that the gradients of temperature, steam, moisture content and the probability of internal overheating are reduced in the material, while reducing energy consumption by $11 \ldots 12 \%$ and improving the thermal and electrical modes of operation of microwave energy sources.

\section{Conclusion}

A physical and mathematical model is developed that describes the interrelated processes of electrodynamics, heat, moisture, and steam exchange under the influence of a microwave field on a wet capillary-porous material in the system of a technological microwave chamber and allows us to study and predict the processes of microwave drying of materials. The research has been carried out and the regularities of the heat and moisture transfer processes under different modes of microwave drying have been established. It is shown that the discrete supply of microwave energy significantly reduces the gradients of temperature, steam and moisture content, while reducing energy consumption by $11 \ldots 12 \%$. The probability of an undesirable spontaneous temperature increase at the end of the drying 
cycle is significantly reduced, and the electrical and thermal conditions of the microwave energy source are improved.

The reported study was funded by RFBR and the government of Volgograd region according to the research project № 19-48-340015 p_a.

\section{References}

1. A V Lykov Theory drying Energy, Moscow p 471 (1968)

2. A.M. Afanas'Ev et al. AIP Conference Proceedings, 2188, 030012 (2019)

3. A.M. Afanas'Ev et al. Journal of Physics: Conference Series, 1546(1), 012075 (2020)

4. J. Sobotka, R.Smolka, MATEC Web of Conferences, 93, 01008 (2016)

5. K. Šuhajda, J. Sobotka, J. Gintar, Advanced Materials Research, 1041, 297-302 (2014)

6. K. Šuhajda et al. Wood Research, 59(3), 389-400 (2014)

7. A. V. Vasilyev et al. Procedia engineering, 150, 312-317. (2016) doi:10.1016/j.proeng.2016.07.017

8. A. Vasilyev et al. IOP Conf Series: Journal of Phisics: Conf. Series, 1050, 012094 (2018) doi: 10.1088/1742-6596/1050/1/012094

9. H. Feng, Y. Yin, J.Tang Food Engineering Reviews, 4(2), 89-106 (2012)

10. V. Tunakova, M. Tunak, P. Tesinova, M. Seidlova, J. Prochazka Textile Research Journal, 90(21-22), 2504-2521 (2020)

11. I. Ganeev, K. Karimov, S. Fayzrakhmanov, I. Masalimov, V. Permyakov, Acta Agriculturae Slovenica, 115(2), 261-271 (2020)

12. Y. Bakhracheva IOP Conf Series: Journal of Phisics: Conf. Series, 1050, 012006 (2018); doi.org/10.1088/1742-6596/1050/1/012006 (2018)

13. Y.Bakhracheva IOP Conference Series: Materials Science and Engineering, 680(1), 012024 (2019)

14. Y. Bakhracheva IOP Conference Series: Materials Science and Engineering, 451(1), 012202 (2018)

15. A.V. Vasilyev et al. Lecture Notes in Mechanical Engineering, 0(9783319956299), 393-399 (2019)

16. S.P. Rudobashta, G.A. Zueva, É.M. Kartashov Journal of Engineering Physics and Thermophysics, 91(1), 227-236 (2018)

17. L.O.Ngkoimani et al. Journal of Physics: Conference Series, 846(1), 012023 (2017) 